\title{
Screening de Lesiones Orales Malignas y Potencialmente Malignas en Funcionarios de Universidad de Valparaíso y Universidad Viña del Mar Durante los Años 2016-2017
}

\author{
Screening of Malignant and Potentially Malignant Oral Lesions in Employee of the \\ University of Valparaíso and the University of Viña del Mar during 2016-2017
}

Karina Cordero T.1; Coral Torres M.2; Paula Anabalón T.2; María José Fernández S.2; Josefina Suzarte R. ${ }^{2}$; Kai Wu T.2; Carolina Asencio O. ${ }^{3}$ \& María Consuelo Salas G. ${ }^{3}$

CORDERO, T. K.; TORRES, M. C.; ANABALÓN, T. P.; FERNÁNDEZ, S. M. J.; SUZARTE, R. J.; WU, K. T.; ASENCIO, O. C. \& SALAS, G. M C. Screening de lesiones orales malignas y potencialmente malignas en funcionarios de Universidad de Valparaíso y Universidad Viña del Mar durante los años 2016 - 2017. Int. J. Odontostomat., 14(2):172-176, 2020.

RESUMEN: El objetivo de este estudio es determinar la prevalencia de lesiones orales malignas y potencialmente malignas (LPM) en funcionarios de la Universidad de Valparaíso y de Viña del Mar durante los años 2016 - 2017. Fueron citados 161 funcionarios, quienes accedieron a una encuesta que evaluaba factores de riesgo de cáncer oral (tabaco y alcohol) y si habían escuchado de la patología, sumado a un examen clínico. De los pacientes examinados, se diagnosticaron 121 lesiones de las cuales 2 fueron diagnosticadas como lesiones potencialmente malignas: Liquen plano y Leucoplasia, con una prevalencia de 1,7\%. El 50,3\% de los pacientes sabía de la existencia de cáncer oral. El Screening es una medida que permite dar a conocer a la población la existencia de cáncer oral y alertarlos sobre la importancia de su examen para su identificación de manera temprana.

PALABRAS CLAVE: detección precoz de cáncer, cáncer oral, desórdenes orales potencialmente malignos.

\section{INTRODUCCIÓN}

El cáncer oral es una de las enfermedades más agresivas del sistema estomatognático, siendo una entidad nosológica que constituye un problema de salud en varios países y con gran interés en disminuir su incidencia (López-Jornet \& De la Mano-Espinosa, 2011). En Chile la mortalidad por cáncer oral y orofaríngeo es del $1 \%$ y la morbilidad corresponde al $1,6 \%$ de todos los cánceres en Chile. Para el año 2010, según diferentes autores, la proporción hombre / mujer varía de 1,3: 1 a 1,4: 1 y la supervivencia a cinco años, de $46 \%$ a $56,9 \%$ (Santelices et al., 2016).

Es conocido también, que existe un gran número de factores de riesgo que son relevantes para el desarrollo de esta enfermedad como el consumo de tabaco y alcohol. En Chile, la ingesta de alcohol tiene una prevalencia mes del $48,9 \%$, situándose en el primer lugar en comparación con los países de la región, consumién- dose una cantidad total de 9,6 litros per cápita al año (Leyton \& Arancibia, 2016). En relación al consumo de tabaco en Chile, el $40,6 \%$ de los mayores de 15 años se declaran fumadores. Estos hábitos nocivos constituyen un importante problema de salud pública a nivel nacional (Ramirez et al., 2015).

Con respecto al virus del papiloma humano (VPH), estudios epidemiológicos indican que la infección oncogénica oral por este virus aumenta de forma significativa respecto a la edad y se evidenció que es mayor en hombres que en mujeres (Chaturvedi et al., 2015), siendo un factor de riesgo de cáncer orofaríngeo.

El carcinoma espinocelular constituye el 92-95 $\%$ de todos los cánceres orales, y la mayoría de ellos es precedido por una lesión precancerosa (Ganesh et al., 2018).

${ }^{1}$ Cirujano Dentista, Especialista en Patología Oral. Profesor Adjunto. Universidad de Valparaíso, Valparaíso, Chile.

${ }^{2}$ Cirujano Dentista, Universidad de Valparaíso. Valparaíso, Chile.

${ }^{3}$ Cirujano Dentista, Universidad de Viña del Mar, Valparaíso, Chile. 
CORDERO, T. K.; TORRES, M. C.; ANABALÓN, T. P.; FERNÁNDEZ, S. M. J.; SUZARTE, R. J.; WU, K. T.; ASENCIO, O. C. \& SALAS, G. M C. Screening de lesiones orales malignas y potencialmente malignas en funcionarios de Universidad de Valparaíso y Universidad Viña del Mar durante los años 2016 - 2017. Int. J. Odontostomat., 14(2):172-176, 2020.

Las lesiones potencialmente malignas son afecciones que preceden a la aparición de cánceres invasivos de la cavidad oral y tienen mayor riesgo de transformación en lesiones malignas (Ganesh et al.; Warnakulasuriya, 2018). El pronóstico del potencial de malignización depende del grado de displasia a nivel histológico, de ahí la importancia de realizar una biopsia (Amagasa et al., 2011). Las técnicas de tamizaje o "Screening" se definen como una intervención estructurada para el cuidado de la salud, diseñada para detectar una enfermedad en un estado latente o asintomático, interrumpiendo la progresión de ésta o facilitando su cura (Monteiro et al., 2015). De esta manera, permite identificar de manera temprana los factores de riesgo, susceptibilidad o la presencia de una lesión tanto potencialmente maligna como maligna, colaborando al diagnóstico precoz y la oportuna derivación. Esto es de suma importancia para mejorar la supervivencia y reducir el retraso en el diagnóstico, ya que, desafortunadamente, casi la mitad de los cánceres orales se detectan en estadios avanzados (III y IV) (Panwar et al., 2014), con tasas de supervivencia a cinco años que se ha mantenido en las últimas décadas (Kumar et al., 2016).

Es de vital importancia que el odontólogo esté entrenado en la detección temprana de cualquier lesión de la cavidad oral, más aún cuando se trata de una lesión con potencial maligno.

El objetivo de esta investigación es determinar la prevalencia de lesiones orales malignas y potencialmente malignas (LPM) en funcionarios de la Universidad de Valparaíso y de Viña del Mar durante los años 2016 2017.

\section{MATERIAL Y MÉTODO}

Se realizó un estudio descriptivo de corte transversal en funcionarios sobre 40 años de la Universidad de Valparaíso y Universidad Viña del Mar, entre enero de 2016 y mayo de 2017. Se reclutaron a los funcionarios a través de un correo electrónico a este grupo, invitándolos a participar en el estudio. Los examinadores fueron calibrados con la investigadora principal, especialista en Patología Oral (Gold Standard). Posteriormente se los citó a un examen odontológico, donde previa firma del consentimiento informado, se recolectaron datos epidemiológicos, nivel de educación, área de desempeño laboral dentro de la universidad, hábitos de tabaco y alcohol y si había escuchado sobre el cáncer oral, pero no si conocía en sí la patología, debido a que conocerla implica una suma de conocimientos teóricos y prácticos sustentados en otros niveles de estudios. A todos los participantes se les examinó en un sillón odontológico, con instrumental de examen y las lesiones orales fueron registradas fotográficamente.

Los datos obtenidos fueron tabulados en el programa Microsoft Office Excel 2010. Se realizaron tablas de frecuencia para las variables cualitativas y las cuantitativas, las que fueron analizadas de acuerdo a las medidas de tendencia central, tales como desviación estándar, promedio y medidas de dispersión. Se utilizó un nivel de significancia de 0,05\%. La investigación fue aprobada por los comités de ética de la Universidad de Valparaíso y Universidad Viña del Mar.

\section{RESULTADOS}

De un total de 161 pacientes examinados (100 pertenecientes a la Universidad de Valparaíso y 61 a la Universidad de Viña del Mar), 57,1 \% correspondieron a mujeres y $42,9 \%$ a hombres. La edad promedio fue de 51 años. Según el nivel de escolaridad, un $46 \%$ tiene enseñanza superior universitaria completa, 30,4 \% es técnico superior, $19,3 \%$ enseñanza media completa y 4,3\% enseñanza básica completa. De acuerdo al tipo de ocupación, un $75,8 \%$ son funcionarios administrativos y docentes, y $24,2 \%$ corresponde a personal auxiliar (Tabla I).

El 50,3\% de los pacientes sabía de la existencia de cáncer oral, la mayor frecuencia se dio en las mujeres, con un $28,6 \%$, mientras que en los varones fue del $21,7 \%$ (Tabla II).

Con respecto a la distribución de hábitos de tabaco y alcohol, un $38,5 \%$ de los pacientes encuestados es fumador. Mientras que un $69,4 \%$ tiene el hábito de beber alcohol (Tabla I). De los pacientes fumadores, sólo 2 de ellos presentaron LPM, y de los que bebían alcohol, 3 presentaron LPM (Tabla III).

Se diagnosticaron 121 lesiones de las cuales 2 fueron diagnosticadas como LPM: Liquen plano y Leucoplasia reticular, con una prevalencia de 1,7\% (Tabla IV).

Otras lesiones detectadas fueron lesiones reaccionales $(55,4 \%)$, trastornos del desarrollo (14 \%), otras lesiones/condiciones $(10,7 \%)$, neoplasia benigna $(7,4 \%)$, lesiones de base inmunitaria $(7,4 \%)$, infecciosas (3,3\%) (Fig. 1). 
CORDERO, T. K.; TORRES, M. C.; ANABALÓN, T. P.; FERNÁNDEZ, S. M. J.; SUZARTE, R. J.; WU, K. T.; ASENCIO, O. C. \& SALAS, G. M C. Screening de lesiones orales malignas y potencialmente malignas en funcionarios de Universidad de Valparaíso y Universidad Viña del Mar durante los años 2016 - 2017. Int. J. Odontostomat., 14(2):172-176, 2020.

Tabla I. Características sociodemográficas y hábitos en pacientes observados en screening.

\begin{tabular}{ll}
\hline Variables & $\begin{array}{c}\text { Frecuencia/ } \\
\text { Porcentaje }\end{array}$ \\
\hline Sexo & \\
Femenino & $92(57,1 \%)$ \\
Masculino & $69(42,9 \%)$ \\
Edad & \\
$40-50$ años & $80(50 \%)$ \\
$51-60$ años & $61(37,8 \%)$ \\
$61-70$ años & $20(12,4 \%)$ \\
Escolaridad & \\
Superior universitaria & $74(46 \%)$ \\
Técnico superior & $49(30,4 \%)$ \\
Enseñanza media & $31(19,3 \%)$ \\
Enseñanza básica & $7(4,3 \%)$ \\
Ocupación & \\
Administrativo/Docente & $122(75,8 \%)$ \\
Personal auxiliar & $39(24,2 \%)$ \\
¿Habían escuchado hablar de cáncer oral? & \\
Sí & $81(50,3 \%)$ \\
No & $80(49,7 \%)$ \\
Fumador & \\
Sí & $62(38,5 \%)$ \\
No & $99(61,5 \%)$ \\
Consumo de aloohol & \\
Sí & $111(69,4 \%)$ \\
No & $50(30,6 \%)$ \\
\hline & \\
\hline & \\
\hline &
\end{tabular}

Tabla II. Relación sobre la pregunta ¿Ha escuchado del cáncer oral?, según sexo.

\begin{tabular}{lccc}
\hline $\begin{array}{l}\text { Ha escuchado } \\
\text { del cáncer oral }\end{array}$ & Femenino & Masculino & Total \\
\hline Sí & $46(28,6 \%)$ & $35(21,7 \%)$ & $81(50,3 \%)$ \\
No & $46(28,6 \%)$ & $34(21,1 \%)$ & $80(49,7 \%)$ \\
Total & 92 & 69 & 161 \\
\hline
\end{tabular}

Tabla III. Presencia de lesión en fumadores y pacientes que beben alcohol

\begin{tabular}{lcc}
\hline & $\begin{array}{c}\text { Presencia } \\
\text { de LPM }\end{array}$ & $\begin{array}{c}\text { Ausencia } \\
\text { de LPM }\end{array}$ \\
\hline Fumador & 2 & 60 \\
No Fumador & 2 & 97 \\
Consumo de OH & 3 & 108 \\
No consumo de $\mathrm{OH}$ & 1 & 49 \\
\hline
\end{tabular}

\section{DISCUSIÓN}

En la presente investigación se estudiaron lesiones malignas y potencialmente malignas orales en funcionarios mayores de 40 años de la Universidad
Tabla IV. Tipo de lesiones orales obtenidas al screening.

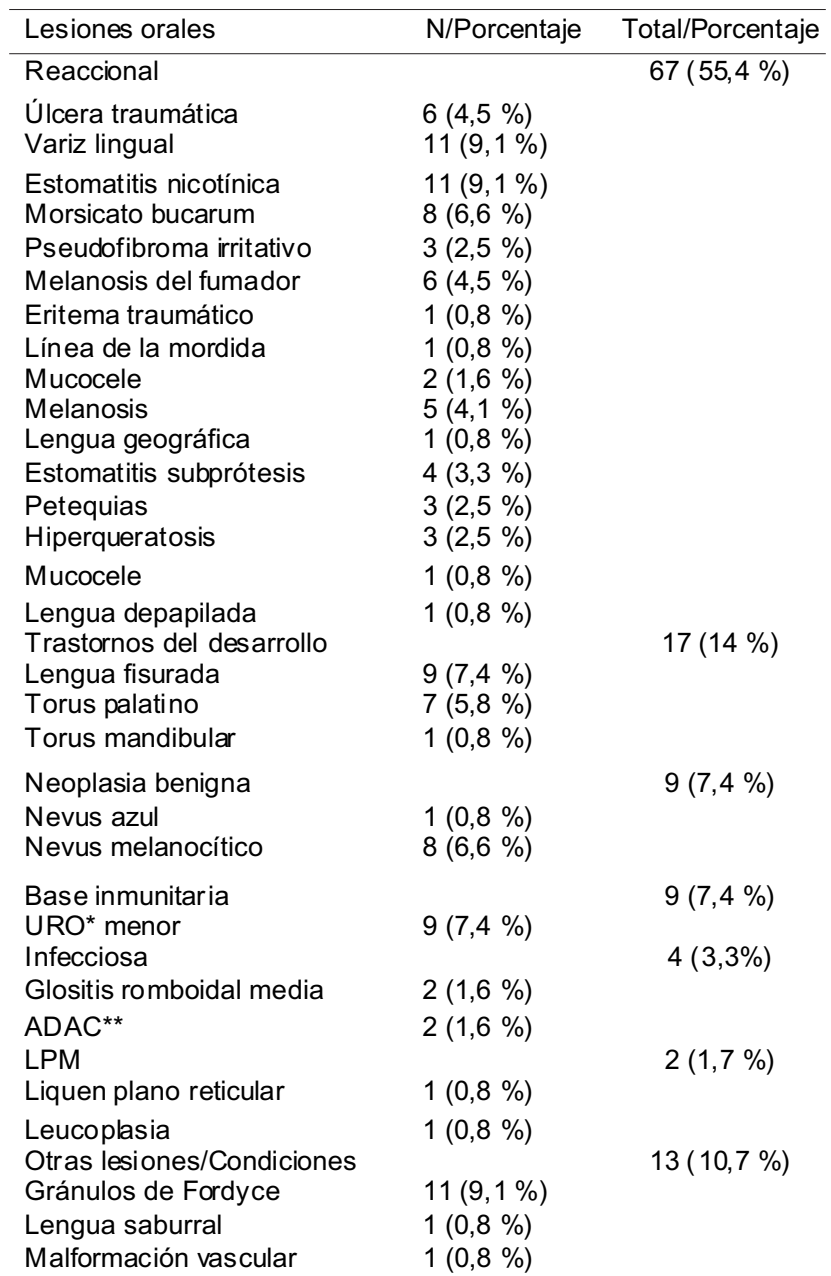

*URO: Úlcera recurrente oral

${ }^{* *}$ ADAC: Absceso dentoalveolar crónico

\section{Clasificación de lesión}

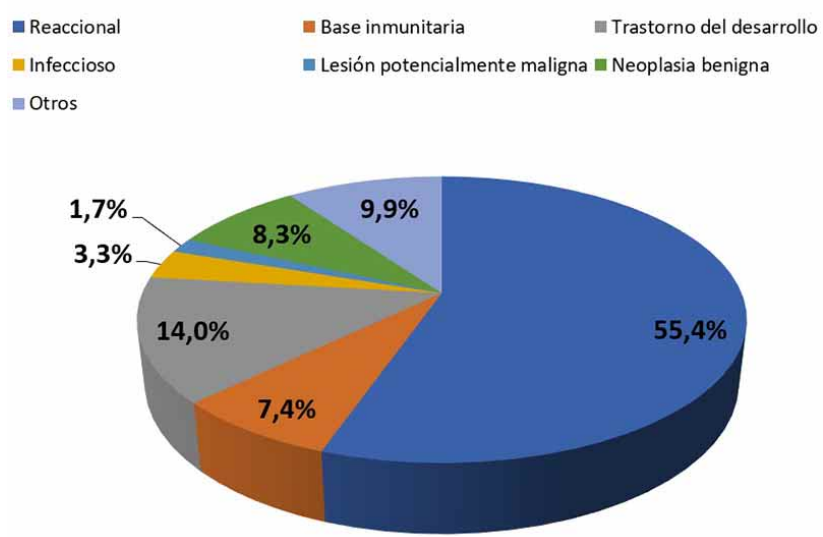

Fig. 1. Clasificación de lesiones orales. 
CORDERO, T. K.; TORRES, M. C.; ANABALÓN, T. P.; FERNÁNDEZ, S. M. J.; SUZARTE, R. J.; WU, K. T.; ASENCIO, O. C. \& SALAS, G. M C. Screening de lesiones orales malignas y potencialmente malignas en funcionarios de Universidad de Valparaíso y Universidad Viña del Mar durante los años 2016 - 2017. Int. J. Odontostomat., 14(2):172-176, 2020.

de Valparaíso y Viña del Mar por un periodo de 2 años (2016 - 2017). Para llevar a cabo esta investigación, se realizó un estudio que nos permitiera conocer la prevalencia de este tipo de lesiones y acercar a la población al conocimiento de la existencia de cáncer oral.

Las LPM más prevalentes en este estudio correspondieron a leucoplasia reticular y liquen plano, siendo la leucoplasia una de las LPM más comunes. Un estudio de Monteiro et al. (2015) durante un programa de screening de cáncer oral en la ciudad de Oporto, Portugal, detectaron 22 lesiones potencialmente malignas, de las cuales un 59,1\% corresponden a leucoplasia y un $40,9 \%$ a liquen plano oral, coincidiendo con nuestro estudio. Se reconocen varias variantes de la leucoplasia y los subtipos clínicos pueden ayudar a determinar el pronóstico hasta cierto punto. La biopsia es esencial para confirmar el diagnóstico clínico provisional y está indicada la derivación oportuna a un especialista (Warnakulasuriya). Los dos pacientes a los que les diagnosticaron LPM fueron derivados a un especialista en Patología Oral para confirmar su diagnóstico mediante una biopsia de la lesión.

La distribución de las lesiones encontradas en este estudio en las personas que declaran consumir tabaco y alcohol es independiente de estos factores de riesgo, lo cual puede deberse a que no son los únicos factores que podrían provocar estas lesiones. Un estudio reciente realizado en Taiwán, indica que pacientes que consumen alcohol tienen un $23 \%$ de riesgo de transformación maligna de las LPM, en comparación con pacientes que no beben (Chuang et al., 2018). Por otra parte, estudios observacionales no prospectivos han señalado una asociación entre el tabaquismo y la leucoplasia oral (Warnakulasuriya \& Ariyawardana, 2016). En un Screening de cáncer oral realizado en la población de Taiwán se señala que fumadores de cigarrillos presentan un riesgo relativo de 2.7 veces de desarrollar leucoplasia oral o cáncer oral (Chuang et al., 2017).

En relación al consumo de tabaco y alcohol en conjunto, se puede decir que la presencia de ambos aumenta aún más la posibilidad de tener una lesión oral premaligna. El riesgo atribuible de cáncer oral debido tanto a tabaco y alcohol se estima que es más del $80 \%$. Los grandes bebedores y fumadores tienen 38 veces más riesgo de desarrollar cáncer que abstemios de ambos productos (Leyton \& Arancibia).
El 50,3\% de los pacientes había escuchado hablar sobre la existencia de cáncer oral. Este porcentaje es bajo al ser comparado con un estudio de Al-Maweri et al. (2017) que indica que el $62,4 \%$ de paciente de Arabia Saudita tenía conocimiento de cáncer oral. Mientras que un $68,2 \%$ y $56,5 \%$, respectivamente, pudieron identificar correctamente el tabaco y el alcohol como factores de riesgo (Al-Maweri et al.). En India, un estudio reciente de Kumar et al. (2018) reportó un conocimiento de cáncer oral de un $92 \%$ de la población encuestada. Este estado educativo se asoció significativamente con un buen conocimiento de la conciencia del cáncer oral y sus factores de riesgo asociados, identificándose los medios de comunicación como la principal fuente de difusión del conocimiento del cáncer oral (Kumar et al., 2018).

Debido a lo anterior, debiese ser pertinente evaluar percepciones y conceptos de cáncer oral, mediante una investigación de tipo cualitativa, que permita conocer aspectos asociados al riesgo, cuidado y prevención del cáncer oral, ya que si bien existe una tendencia entre las personas a saber más sobre la existencia de esta patología, la prevalencia también tiende al alza, incluso entendiéndose cuales son algunos de los factores de riesgos asociados, no obstante es una patología de la cual no existe conciencia ni suficiente visibilidad en la población.

El screening sirve como una herramienta de detección temprana de lesiones malignas y potencialmente malignas, así como el llamado al autocuidado y autoexamen oral. Debido a que actualmente no existe un sello patognomónico molecular o histopatológico que pueda predecir la transformación maligna de trastornos potencialmente malignos, el análisis de los aspectos clínicos de estas lesiones sigue siendo la mejor manera de controlar y prevenir el desarrollo de cáncer oral (Ganesh et al., 2018).

\section{CONCLUSIÓN}

A pesar de encontrar una baja prevalencia de LPM en este grupo, el screening resultó beneficioso para esta población, ya que fomentó el conocimiento del cáncer oral, así como el autocuidado y autoexamen oral. Nuestros resultados podrían servir como modelo para el diseño de nuevas estrategias para campañas de detección de cáncer oral a nivel nacional, y así establecer una relación significativa entre LPM y factores de riesgo, para un diagnóstico temprano y tratamiento certero. 
CORDERO, T. K.; TORRES, M. C.; ANABALÓN, T. P.; FERNÁNDEZ, S. M. J.; SUZARTE, R. J.; WU, K. T.; ASENCIO, O. C. \& SALAS, G. M C. Screening of malignant and potentially malignant oral lesions in employees of the University of Valparaíso and the University of Viña del Mar during 2016 - 2017. Int. J. Odontostomat., 14(2):172-176, 2020.

ABSTRACT: The objective of this study is to determine the prevalence of malignant and potentially malignant oral lesions (PML) in University of Valparaíso and Viña del Mar employees, during the years 2016 - 2017. We cited 161 employees, who accessed a survey evaluating risk factors for oral cancer (tobacco and alcohol) and if they had heard of the disease. In addition, a clinical examination was carried out. Of the patients examined, 121 lesions were diagnosed, of which 2 were diagnosed as potentially malignant lesions: Lichen planus and Leukoplakia, with a prevalence of $1.6 \% .50 .3 \%$ of patients knew of the existence of oral cancer. Epidemiological designs are needed to better establish causality between risk factors and malignant or, potentially malignant lesions.

KEY WORDS: early detection of cancer, oral cancer, oral potentially malignant disorders.

\section{REFERENCIAS BIBLIOGRÁFICAS}

Al-Maweri, S. A.; Al-Soneidar, W. A.; Dhaifullah, E.; Halboub, E. S. \& Tarakji, B. Oral cancer: awareness and knowledge among dental patients in Riyadh. J. Cancer Educ., 32(2):308-13, 2017.

Amagasa, T.; Yamashiro, M. \& Uzawa, N. Oral premalignant lesions: from a clinical perspective. Int. J. Clin. Oncol., 16(1):5-14, 2011.

Chaturvedi, A. K.; Graubard, B. I.; Broutian, T.; Pickard, R. K. L.; Tong, Z. Y.; Xiao, W.; Kahle, L. \& Gillison, M. L. NHANES 2009-2012 Findings: association of sexual behaviors with higher prevalence of oral oncogenic human papillomavirus infections in U.S. men. Cancer Res., 75(12):2468-77, 2015.

Chuang, S. L.; Su, W. W. Y.; Chen, S. L.; Yen, A. M. F.; Wang, C. P.; Fann, J. C. Y.; Chiu, S. Y. H.; Lee, Y. C.; Chiu, H. M.; Chamg, D. C.; et al. Population-based screening program for reducing oral cancer mortality in 2,334,299 Taiwanese cigarette smokers and/or betel quid chewers. Cancer, 123(9):1597-609, 2017.

Chuang, S. L.; Wang, C. P.; Chen, M. K.; Su, W. W. Y.; Su, C. W.; Chen, S. L. S.; Chiu, S. Y. H.; Fann, J. C. Y. \& Yen, A. M. F. Malignant transformation to oral cancer by subtype of oral potentially malignant disorder: a prospective cohort study of Taiwanese Nationwide Oral Cancer Screening Program. Oral Oncol., 87:5863, 2018.

Ganesh, D.; Sreenivasan, P.; Öhman, J.; Wallström, M.; Braz-Silva, P. H.; Giglio, D.; Kjeller, G. \& Hasséus, B. Potentially malignant oral disorders and cancer transformation. Anticancer Res., 38(6):32239, 2018.

Kumar, M.; Nanavati, R.; Modi, T. G. \& Dobariya, C. Oral cancer: Etiology and risk factors: A review. J. Cancer Res. Ther., 12(2):45863, 2016.

Kumar, S.; Kumari, P.; Gupta, R.; Singh, S. K.; Sinha, S.; Mehta, P. \& Chourasia, S. K. Knowledge and awareness of oral cancer and impact of pictorial warnings on the willingness to quit tobacco in young tobacco consumers in India. Int. J. Adolesc. Med. Health, 2018. doi: 10.1515/ijamh-2018-0185 [Online ahead of print]
Leyton, F. F. \& Arancibia, P. El Consumo de Alcohol en Chile: Situación Epidemiológica. Santiago de Chile, Ministerio de Salud, SENDA Ministerio del Interio y Seguridad Pública, Gobierno de Chile, 2016. Disponible en: http://www.senda.gob.cl/wp-content/uploads/ media/estudios/otrosSENDA/2016_Consumo_Alcohol_Chile.pdf

López-Jornet, P. \& De la Mano-Espinosa, T. The efficacy of direct tissue fluorescence visualization in screening for oral premalignant lesions in general practice: an update. Int. J. Dent. Hyg., 9(2):97-100, 2011.

Monteiro, L. S.; Salazar, F.; Pacheco, J. J.; Martins, M. \& Warnakulasuriya, S. Outcomes of invitational and opportunistic oral cancer screening initiatives in Oporto, Portugal. J. Oral Pathol. Med., 44(2):145-52, 2015.

Panwar, A.; Lindau, R. \& Wieland, A. Management for premalignant lesions of the oral cavity. Expert Rev. Anticancer Ther., 14(3):34957,2014

Ramirez, V.; Vásquez-Rozas, P. \& Ramírez-Eyraud, P. Mortalidad por cáncer oral y faríngeo en Chile, años 2002-2010. Rev. Clin. Periodoncia Implantol. Rehabil. Oral, 8(2):133-8, 2015.

Santelices, C. M. J.; Cárcamo, I. M.; Brenner, A. C. \& Montes, F. R. Oral cancer: review of the Chilean literature. Rev. Med. Chile, 144(6):758-66, 2016.

Warnakulasuriya, S. \& Ariyawardana, A. Malignant transformation of oral leukoplakia: a systematic review of observational studies. J. Oral Pathol. Med., 45(3):155-66, 2016.

Warnakulasuriya, S. Clinical features and presentation of oral potentially malignant disorders. Oral Surg. Oral Med. Oral Pathol. Oral Radiol., 125(6):582-90, 2018.

Dirección para correspondencia:

Karina Cordero T.

Cirujano Dentista

Especialista en Patología Oral

Profesora Adjunto

Universidad de Valparaíso

Valparaíso

CHILE

Email: karina.cordero@uv.cl

Recibido : 19-08-2019

Aceptado: 05-11-2019 